\title{
NIR studies of galactic giant HII regions
}

\author{
A. Damineli ${ }^{1}$, R. D. Blum ${ }^{2}$, E. Figuerêdo ${ }^{1}$ and P. S. Conti ${ }^{3}$ \\ ${ }^{1}$ IAGUSP, University of São Paulo, 05508-900, Brazil \\ email: damineli@astro.iag.usp.br \\ ${ }^{2}$ Cerro Tololo Interamerican Observatory, Casilla 603, La Serena, Chile \\ email: Blum@noao.edu \\ ${ }^{3}$ JILA, University of Colorado, Campus Box 440, Boulder, CO, 80309, USA \\ email: Pconti@jila.colorado.edu
}

\begin{abstract}
We present results from our ongoing survey of Galactic Giant HII Regions in the near-infrared. The luminosity function indicates that the IMF is uniform, independent of the galactocentric distance and compatible with Salpeter (1955) slope. Distances measured by the spectroscopic parallax method are systematically smaller than kinematic distances derived from radio techniques. As a consequence, the number of ionizing photons and the star formation rate is much lower than that derived from rotation models. Although the luminosities of the giant HII regions obtained from this method are lower than by other methods, the morphological type of the Milky Way is well in line of previous results, close to Sbc or Sc types.
\end{abstract}

Keywords. surveys, stars: distances, stars: early type, stars: formation, (ISM): HII regions, Galaxy: disk, infrared: general

\section{Introduction}

Massive stars $\left(M>8 \mathrm{M}_{\odot}\right)$ are rare in the local Universe because of three factors: a) in a star formation event, the number of objects decreases steeply as the mass increases; b) massive star formation is rare in the local Universe; c) the lifetime of an O-type star is only a few million years. As the Universe gets older, the proportion of massive objects gets extremely low. In a Galaxy like the Milky Way, for every billion stars, only one is more massive than $\mathrm{M}>60 \mathrm{M}_{\odot}$. In such an environment, the opportunities to test theories for high mass stars are meager. The result is a poor knowledge of formation process and evolution of objects in the upper HR diagram. In spite of that, Galactic objects offer the best chances to be studied at high spatial and spectral resolution.

The study of massive star clusters in the Milky Way is important to address a number of questions. What is the precise kind of spiral Galaxy we live in? What is the number and position of the spiral arms? What is the current star formation rate (SFR)? How the slope of the initial mass function (IMF) changes as function of the galactocentric distance? What is the mechanism of mass accretion for high mass stars? What are the observational properties of the different formation phases, from a pre-stellar contracting core to a naked photosphere? Giant HII regions (GHIIRs) - defined as those powered by one O3V star (or ten O7V) - are relevant to study massive star formation in our Galaxy. As can be observed in other Galaxies, they trace very well the spiral arms.

Our Sun is in the Galactic plane (GP), a disadvantageous position, because the bulk of massive stars concentrates in a thin strip \pm 1 degree each side of the GP. This causes source confusion and moreover, the obscuration along the GP is huge, limiting the observations in the optical window to distances of $<5 \mathrm{kpc}$. The interstellar extinction decreases toward longer wavelengths, but the photospheric emission of hot stars also decreases. At wavelengths larger than $2 \mu \mathrm{m}$, the emission from hot dust grows steeply. The K-band 
$(2-2.5 \mu \mathrm{m})$ is strategic to beat the extinction and, at the same time, to display spectral lines (HeII, HeI, NIII, CIV and HI) useful to classify OB-type stars. Detectors sensitive to near-infrared (NIR) radiation $(1-2.5 \mu \mathrm{m})$ have been increasing in size (1-4 Mpixel) and improving in response uniformity through the array, approaching the performance of CCDs in the optical window. The fact that the K-band is in the Jeans tail of the energy distribution for hot stars, makes their color indices degenerated. This apparent disadvantage results to be useful, since the intrinsic color indices are almost the same for all $\mathrm{OB}$ stars $(\mathrm{H}-\mathrm{K})_{0}=-0.04$. The measured color index is essentially the color excess due to the interstellar reddening (in the absence of circumstellar emission). Moreover, the reddening law is largely insensitive the reddening law $\left(\mathrm{A}_{\lambda}=\mathrm{A}_{K}[2.2 / \lambda]^{1.75}\right)$ resulting in $\mathrm{AK}=1.6 \times \mathrm{E}(\mathrm{H}-\mathrm{K}), \mathrm{A}_{H}=1.7 \times \mathrm{A}_{K}$ and $\mathrm{A}_{J}=2.7 \times \mathrm{A}_{K}$. The interstellar extinction for stars near the Galactic center $(\mathrm{GC})$ is $\mathrm{A}_{V} \sim 30\left(\mathrm{~A}_{K} \sim 3\right)$. Since GHIIRs form at the borders of molecular clouds (MC), they are subject to substantial local obscuration that adds up to the interstellar reddening. In this way, very young OB stars are always seen under $\mathrm{A}_{K} \sim 2-5$, even for directions far from the GC. On top of the large reddening, the crowding limits the assessment of the stellar content of clusters powering GHIIRs. The practical limit to study such clusters is $\mathrm{d}<10 \mathrm{kpc}$, leaving the far side of our Galaxy out of reach of the present day instruments. Only ELT-class telescopes and high order AO correction can improve this situation.

\section{Selection criteria of our GHIIR sample}

Our aim is to study young massive stellar clusters is to explore the crossroads between the structure of our Galaxy and the physics of formation of massive stars. A GHIIR is defined as being powered by at least an O3V star, what means a luminosity NLyC $>0.43 \times 10^{50}$ photons per second in the Lyman continuum. In reality, due to the nature of the IMF, we expect that a GHIIR is powered by at least a dozen O-type and tens B-type stars. The NLyc is measured from the radio luminosity of the source. Since the interstellar medium (ISM) is highly transparent to radio waves, the HII region luminosity is easily derived when the distance is known. However, the correct assessment of NLyC is less straightforward than it looks at first glance. Some LyC photons may not produce ionization because are destroyed by dust grains or leakage through the cloud. As a matter of fact, the HII radio luminosity indicates only a lower limit to the NLyC emitted by the stellar cluster. Distance determination is a major problem. It is usually derived by inputting the radial velocity of the gas into a rotation model. Radial velocity measurements of radio recombination lines are pretty accurate. The problem arises when interpreting then as Galactic motion. On one hand, rotation models can't be checked by independent methods throughout most of the Galaxy. On the other hand, HII regions can depart from pure Galactic rotation, as they are formed at the borders of MCs. Collisions between MCs result in linear and angular momenta that add to the galactic rotation. Moreover, as the gas is heated by the ionizing stars, it escapes from the HII region to the ISM in champagne flows with velocities up to $10 \mathrm{Km} \mathrm{s}^{-1}$. Brand \& Blitz (1993) report local deviations from rotation curve of up to $40 \mathrm{Km} \mathrm{s}^{-1}$ in the outer Galaxy. There are additional problems involved in kinematic distances. The rotation model is degenerate for positions inside the orbit of the Sun around the GC. The solution of the equation of the observed motion result in two values: the near and far distances. In principle, there is a way to break the near/far ambiguity by measuring radial velocities of absorption lines in front of the source. For the particular direction of the Galactic center, however, the model is completely degenerate: the radial velocity is zero for all distances. In 
practice, due to measurement uncertainties and non-rotational velocities, kinematic distances close to the GC line-of-sight are unreliable.

Taking into account the above considerations, we need to include targets in our sample that do not fit the simple requirement of NLyC $>0.43 \times 10^{50} \mathrm{ph} \mathrm{s}^{-1}$. For lines-of-sight close to the GC, any single obscured HII region is a potential GHIIR candidate. For other sources toward the inner solar circle, we need to include as candidates, all HII regions that have unreliable distance determinations, even if they look to be significantly fainter than the above limit. Since the star formation activity is large in the inner Galaxy, we may end up with too many candidates. Fortunately, there is a simple way to reject the most obvious false candidates, as follows. We start with a Galactic radio source catalogue, like the one published by Russeil (2003), which reports kinematic distances. To check if some particular radio source corresponds to an active star forming region, we search for its counterpart in MSX satellite catalogue. Young HII regions contain enshrouded OB stars that are luminous mid-infrared sources and can be seen everywhere in the Galaxy, since the ISM is very transparent at these wavelengths. A look in the 2MASS survey can give a clue to the distance of the HII region. For example, a nearby HII region displays clearly the stellar cluster plus the ionized nebula in all the JHK bands. As the distance increases, both components are dimmed by dust extinction. At an intermediate distance, both the nebula and the cluster are still visible in the $\mathrm{K}$-band, but faint or undetected in the J-band. Stars dim more rapidly $\left(\sim 1 / d^{2}\right)$ than the associated nebula $(\sim 1 / d)$, in order that HII regions at $\mathrm{d}>6 \mathrm{kpc}$ toward the inner Galaxy are seen as faint nebulae in the 2MASS K-band images, devoid of stars. A visual inspection in the 2MASS images, following such a procedure, enabled us to break the near/far ambiguity and to reject wrong distance assignments for several sources in radio catalogues. We have examined 22 sources reported in Russeil (2003). For 16 sources assigned to near distance, 14 were confirmed, 1 is at intermediate distance and 1 at far distance. For 4 sources assigned to far distance, 2 were confirmed and 2 were found to be near. For 2 sources assigned to intermediate both were found to be near. In this way $\sim 30 \%$ of the sources are at wrong distance, what compares well with that reported by Bik (2004). This author reports spectroscopic parallaxes for 38 sources $\sim 40 \%$ of which have distances in disagreement with the kinematic distance. We revised the list of Galactic GHIIRs presented by Conti \& Crowther (2004) by using the procedure described above and ended up with 60 GHIIR candidates. For some of them, we derived spectroscopic parallax distances, as discussed below.

\section{Photometry and Spectroscopy}

In order to select targets for follow up spectroscopy, we have obtained deep images in the JHK bands at $4-\mathrm{m}$ class telescopes (CTIO and Kitt Peak). After extracting magnitudes through PSF photometry, we construct color-color (CCD) and color-magnitude (CMD) diagrams. The CCD plot has two regions, one containing normal stars and another, objects with excess emission. Normal stars follow a narrow band corresponding to pure interstellar reddening. Candidates to naked O-type stars are selected inside this band, taking into account the typical reddening of the cluster. The spectral classification uses the scheme designed for the K-band by Hanson, Conti \& Rieke (1997) and is accurate within 1-2 spectral types. The luminosity class can't be derived from low resolution spectra, but most HII regions are young enough to have OB stars close to the ZAMS, leading to reliable absolute magnitudes. The spectroscopic parallax distance is derived by comparing the absolute and the apparent (derredned) magnitude. 
Table 1. Distances of HII regions measured by kinematic and spectrophotometric methods

\begin{tabular}{lllll}
\hline Ident. & $\begin{array}{l}\mathrm{K}_{\text {dist }} \\
\mathrm{kpc}\end{array}$ & $\begin{array}{l}\text { ref } \\
\mathrm{SPP}_{\text {dist }}\end{array}$ & ref \\
$\mathrm{kpc}$ & \\
\hline M8 & 2.8 & 1 & 0.6 & 3 \\
W31 & 4.5 & 2 & 3.4 & 4 \\
M17 & 2.4 & 1 & 1.3 & 5 \\
W42 & 11.5 & 1 & 2.2 & 6 \\
W43 & 6.2 & 1 & 4.3 & 7 \\
W49A & 11.8 & 2 & 8.4 & 3 \\
W51A & 5.5 & 1 & 5.7 & 8 \\
NGC3576 & 3.1 & 1 & 0.6 & 3 \\
NGC3603 & 7.9 & 1 & 6.0 & 9 \\
G298.9-0.4 & 10.4 & 1 & 3.0 & 3 \\
G333.1-0.4 & 3.5 & 1 & 2.6 & 10 \\
\hline
\end{tabular}

References: (1)Russeil (2003); (2)Kuchar \& Clark (1997); (3)Bik (2004); (4)Blum et al. (2001); (5)Hanson et al. (1997); (6)Blum et al. (2000); (7)Blum et al. (1999); (8)Figuerêdo et al. (2005b); (9) Stolte et al. (2004); (10) Figuerêdo et al. (2005a)

In addition to the luminosity class, there are two sources of uncertainty that affect the distance: a) the scale of absolute magnitudes has a scatter of 0.7 mag for O-type stars; b) unresolved companions may decrease the apparent magnitude by up to 0.4 mag. However, the distance can be better constrained as multiple spectra accumulates.

\section{Preliminary Results}

We have so far analyzed NIR data from 6 HII regions. This represents only $10 \%$ of our target list and is inadequate to produce statistically significant results. However, the emerging scenario is worth being discussed.

\subsection{The universality of the IMF}

Massey et al. (1995) and other authors have discussed the IMF of young massive clusters from the Magellanic Clouds and the external parts of the Milky Way. They find a universal IMF slope, compatible with Salpeter (1955). There are expectations that the IMF could be flatter in regions of higher metallicity, like that believed to exist in the inner Galaxy. Figer et al. (1999) report a slope $\alpha=-0.9$ for the Arches cluster, close to the GC. A slope flatter than Salpeter $(\alpha=-1.35)$ implies in larger number of massive stars. Some authors claim that this is the case for starburst galaxies. Is the Arches cluster a special case, or a general rule in the inner Galaxy? Our sample is relevant to such a study, since it contains clusters in wide range of Galactocentric distances. The K-band luminosity function (KLF) is very uniform over the sample we've observed, indicating that the IMF also is uniform. Translating the KLF into IMF involves many problems, like binarity and excess emission. Within the uncertainties, the IMF slopes we have derived are compatible with Salpeter slope. Our results are in line with those that point to an universal IMF slope.

\subsection{Star Formation Rate and the Hubble Type of the Milky Way}

The integrated NLyC in Conti \& Crowther (2004) list is $2.3 \times 10^{52} \mathrm{ph} \mathrm{s}^{-1}$, what corresponds to the NLyC from 5676 O7V stars. The sample presented in Table 1, that has distances measured by spectroscopic parallax, amounts to $38 \%$ of the NLyC reported by Conti \& Crowther (2004), based on kinematic distances. If we assume that the same reduction factor applies to the remaining HII regions not yet measured by spectroscopic 


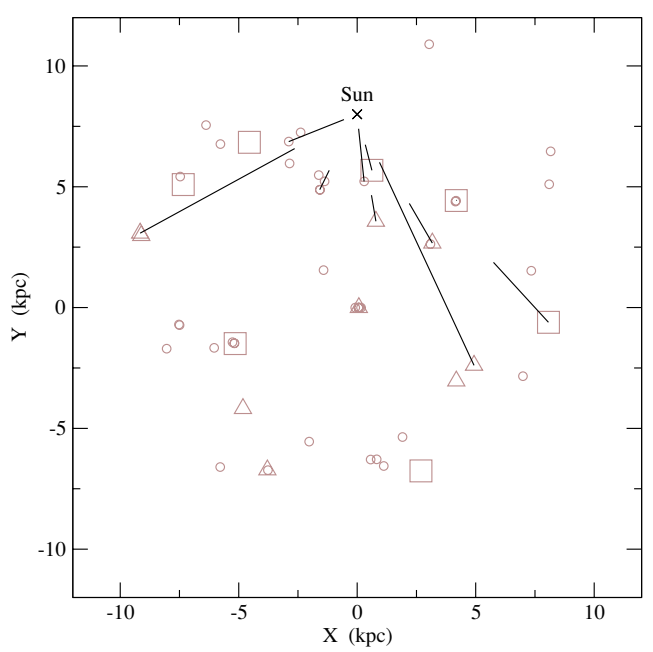

Figure 1. Map of Galactic GHIIRs. Open symbols indicate the galactocentric distances and luminosities derived by kinematic distances from Conti \& Crowther (2004). Solid lines indicate displacements corresponding to the new distances, as measured by spectroscopic parallaxes

parallax (SPP), the integrated number of ionizing photons in the whole Galaxy would be NLyC $\sim 9 \times 10^{51} \mathrm{ph} \mathrm{s}^{-1}$, what is equivalent to 2157 O7V stars.

The star formation activity is correlated with the Hubble morphological type. We can have a guess of the Milky Way morphological type by translating the NLyC in $\mathrm{H} \alpha$ luminosity and comparing with external galaxies (Smith \& Kennicutt 1989). The case B for a radiation bounded HII region at 10,000 K predicts (Brocklehurst 1971):

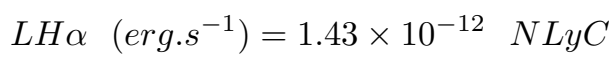

We converted the $\mathrm{NLyC}\left(10^{51.91}\right)$ to equivalent $\mathrm{LH} \alpha\left(10^{40.07}\right)$ for the average of the three most luminous HII regions with reliable distances: W49A, M17 and NGC3603. When adopting $\mathrm{M}_{B}=-20.2$ for the Milky Way, we see that our Galaxy is a Sbc-Sc, in agreement with Smith \& Kennicutt (1989) and results by other authors, based on different criteria. We used updated distances in the list of Conti \& Crowther (2004) and derived the luminosity function (LF) of NLyC for Galactic HII regions. The slope is $\alpha=-2.0$ as compared to $\alpha=-2.2$ derived by Smith \& Kennicutt (1989). Those authors reported $\alpha=-2.0$ for Sbc-Sc Galaxies.

Although the SPP distances disagree from the kinematic method, the morphological type of our Galaxy remains in thee same range. The disagreement is in the location and number of spiral arms, in addition to the SFR of specific HII regions. In order to present a major revision of the spiral structure of the Milky Way, we need to increase the number of SPP distances measured for each cluster and observe a larger sample of HII regions.

\section{Acknowledgements}

A.D. and E.F. thanks to IAU and FAPESP Foundation for financial support.

\section{References}

Bik, A. 2004, PhD thesys

Blum, R.D., Damineli, A., \& Conti, P.S. 1999, AJ 117, 1392

Blum, R.D., Conti, P.S., \& 2000, Damineli, A., AJ 119, 1860 
Blum, R.D., Damineli, A., \& Conti, P.S. 2001, AJ 121, 3149

Conti, P.S. \& Crowther, P.A. 2004, MNRAS 355,899

Figer, D., F., Kim, S.S., Morris, M., Serabyn, E., Rich, R.M., \& McLean, I.S. 1999, ApJ 525, 750

Figuerêdo, E., Blum, R.D., Damineli, A., \& Conti, P.S. 2005, AJ 129, 1523

Figuerêdo, E., Blum, R.D., Damineli, A., \& Conti, P.S. 2005, in preparation

Hanson, M.M., Howartyh, I.D., \& Conti, P.S. 1997, ApJ 489, 698

Kuchar, T.A. \& Clark, F.O. 1997, APJ, 488, 224

Massey, P., Johnson, K.E., \& DeGioia-Eastwood, K. 1995, ApJ 454, 151

Russeil, D. 2003, A\&̈A 397, 133

Salpeter, E.E. 1955, ApJ 121, 161

Smith, T.R. \& Kennicutt, R. 1989, PASP 101, 649

Stolte, A., Brandner, W., \& Brandl, B.K. 2004, AJ 128, 765 